\title{
Vagal Nerve Block for Improvements in Glycemic Control in Obese Patients with Type 2 Diabetes Mellitus: Three-Year Results of the VBLOC DM2 Study
}

\author{
Miguel F Herrera ${ }^{1}$, James Toouli ${ }^{2}$, Bård Kulseng ${ }^{3}$, Roy Brancatisano ${ }^{4}$, Lilian Kow ${ }^{2}$, Juan P Pantoja ${ }^{1}$, \\ Gjermund Johnsen ${ }^{3}$, Anthony Brancatisano ${ }^{4}$, Katherine S Tweden ${ }^{5 *}$, Jonathan J Waataja ${ }^{6}$, Charles J \\ Billington $^{7}$, Scott A Shikora ${ }^{8}$
}

\author{
${ }^{1}$ Instituto Nacional de al Nutricion, Salvador Zubrian (INNSZ), Vasco de Quiroga 15, Tlalpan, 1400 Mexico City, DF, Mexico \\ ${ }^{2}$ Adelaide Bariatric Center, Flinders Private Hospital, Suite 502/Level 5, Bedford Park, SA, 5041, Australia \\ ${ }^{3}$ Center for Obesity, St. Olavs Hospital, Olav Kyrres Gate 6, 7006, Trondheim, Norway \\ ${ }^{4}$ Institute of Weight Control, 17-19 Solent Circuit, Norwest Business Park, Baulkham Hills, NSW 2153, Australia \\ ${ }^{5}$ EnteroMedics Inc, 2800 Patton Road, St. Paul, MN 55113, USA \\ ${ }^{6}$ University of Minnesota, 321 Church Street SE, Minneapolis, Minnesota, 55455, USA \\ ${ }^{7}$ University of Minnesota, Minneapolis, Minnesota Veterans' Administration Medical Center, One Veterans' Drive, Minneapolis, \\ MN 55417, USA \\ ${ }^{8}$ Brigham and Women's Hospital, 75 Francis Street, Boston MA 02115, USA
}

*Corresponding author: Katherine Tweden, PhD. Entero Medics Inc, 2800 Patton Road, St. Paul, MN 55113,

E-mail: ktweden@enteromedics.com

\begin{abstract}
Background: The VBLOC DM2 study demonstrated that intermittent electrical vagal blocking (vBloc therapy) was safe among subjects with obesity and type 2 diabetes mellitus (DM2) and led to clinically meaningful improvement in weight loss and glycemic parameters at 2 years. Sustainability of these responses at three years is reported here.

Methods: VBLOC DM2 is a prospective, observational study of 28 subjects with DM2 and body mass index (BMI) between 30 and $40 \mathrm{~kg} / \mathrm{m}^{2}$. Safety and changes in weight, glycemic parameters and other risk factors with vBloc therapy are assessed. Mixed models are used to report continuous outcome variables.

Results: After three years of therapy, mean percentage of excess weight loss was $21 \%$ (95\% CI, 14 to 28 ) or $7 \%$ total body weight loss (95\% CI, 5 to 9). Hemoglobin $A_{1 c}$ decreased by a mean of 0.6 percentage points (95\% CI, 0.2 to 1.0$)$ from a baseline of $7.8 \%$. Fasting plasma glucose declined by a mean of $18 \mathrm{mg} / \mathrm{dL}(95 \% \mathrm{CI}, 2$ to 34$)$ from a baseline of $151 \mathrm{mg} / \mathrm{dL}$. The most common adverse events continued to be heartburn, constipation and neuroregulator site pain which were mostly mild to moderate in severity as noted in earlier reports.

Conclusions: Three years of treatment with vBloc therapy resulted in durable improvements in weight loss and glycemic control. vBloc was shown to have favorable safety through 3 years.
\end{abstract}

Received date: November 28, 2016

Accepted date: January 10, 2017

Published date: January 24, 2017

Citation: Tweden, K.S., et al. Vagal Nerve Block for Improvements in Glycemic Control in Obese Patients with Type 2 Diabetes Mellitus: Three-Year Results of the VBLOC DM2 Study. (2017) J Diab Obes 4(1): $1-6$.

DOI: $10.15436 / 2376-0494.17 .1250$

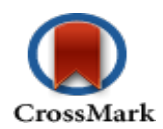

Keywords: Obesity; vBloc; Type 2 diabetes mellitus

Copyrights: (C) 2017 Tweden, K.S. This is an Open access article distributed under the terms of Creative Commons Attribution 4.0 International License. 


\section{Introduction}

It is estimated that there are 347 million people worldwide with $\mathrm{DM} 2^{[1]}$, and its prevalence will likely continue to grow $^{[2]}$. Recent data from the Centers for Disease Control and Prevention estimate that the lifetime risk for acquiring DM2 has increased dramatically in the last two decades to $40.2 \%$ for men and $39.6 \%$ for women ${ }^{[3]}$. Obesity, defined as BMI over $30 \mathrm{~kg} / \mathrm{m}^{2}$, has been acknowledged as the most significant risk factor for developing DM2. Multiple studies and converging views of major societies on the treatment of diabetes have strongly suggested that weight loss (of as little as $2.5 \mathrm{~kg}$ ) significantly reduces the chances of developing DM2 $2^{[4,5]}$.

Risk of potential complications and permanent anatomical alter ations make bariatric surgery an option of last resort for many obese patients with DM2 even though current bariatric surgical interventions such as sleeve gastrectomy, gastric bypass, and biliopancreatic diversion have been shown to have a significant effect on glycemic control and diabetes ${ }^{[6]}$. Diabetes drugs are efficacious treatments for most patients; however, as the disease progresses, patients may require an expensive drug regimen, and often need injectable insulin. The long-term cardiovascular safety profile of these medications is unclear. A weight loss treatment that is durable but less risky than conventional bariatric weight loss surgery would be an appealing option for many patients with obesity who have DM2.

The vagus nerve has long been known to play an important role in food intake and weight regulation. It has been shown that vagotomy induces weight $\operatorname{loss}^{[7]}$. However, this procedure is irreversible, it can cause unwanted side effects and compensatory mechanisms can evolve over time ${ }^{[8]}$. A method of controlled intermittent vagal blockade would be desirable. To address this, a technology was created which uses electrical signals to temporarily and reversibly block conduction through the anterior and posterior sub-diaphragmatic vagal trunks (vBloc therapy).

A randomized, sham-controlled, double-blind, clinical trial of vBloc therapy for moderate to severe obesity in 239 participants showed statistically superior weight loss compared to a sham control with a low rate of complications at one year ${ }^{[9]}$. Recently, it was reported that these results were durable through 2 years and correspondingly demonstrated approximately $50 \%$ remittance of pre-diabetes and metabolic syndrome among participants who presented with the syndromes at baseline ${ }^{[10]}$. Participants also reported sustained significant improvements in quality of life and $50 \%$ reduction in their hunger compared to baseline ${ }^{[10]}$. The VBLOC DM2 study is a prospective feasibility study during which 28 obese participants with DM2 received vBloc therapy. Clinically significant reductions in weight were demonstrated through two years of this trial ${ }^{[11]}$. Of note, improvements in glycemic parameters were observed within weeks of vBloc therapy initiation ${ }^{[12]}$ and were maintained through two years. Given the importance of sustained improvement in glycemic parameters for the diabetic patient, the major focus of this report is to present data through three years.

\section{Materials and Methods}

\section{Study Design}

The VBLOC DM2 study is a prospective, open-label, single-arm investigation with the objective of evaluating the safety and efficacy of vBloc therapy in participants with obesity and DM2. There were no formal endpoints for this investigation and it was not powered to detect any effect size. The sample size of 28 subjects was deemed sufficient to establish performance of the study device and to compare performance results with those from previous investigations based on the precision of the $\%$ EWL estimate. Precision is defined as the half-width of the confidence interval surrounding the mean \%EWL. Assuming a standard deviation of 18 units, 28 subjects provide an estimate of $\%$ EWL with $7 \%$ precision (with $95 \%$ confidence).

Efficacy measures of interest were improvements from baseline in percentage excess weight loss (\%EWL), BMI, fasting plasma glucose and hemoglobin $\mathrm{A}_{1 \mathrm{c}}\left(\mathrm{HbA}_{1 \mathrm{c}}\right)$. Changes in systolic and diastolic blood pressure were measured also, although participants with hypertension were not prospectively enrolled. All adverse events (AEs) that occurred in the trial were captured. The clinical site investigators attributed the AEs as either related or not related to the device, procedure, therapy algorithm or unknown and recorded their severity as mild, moderate or severe. An independent Clinical Events Committee adjudicated the relatedness of each serious adverse event (SAE). Ethics committee approval was obtained at all investigative sites prior to conduct of the study. Informed consent was obtained from all individual participants included in the investigation prior to their participation in the study. Five sites from Australia, Mexico, Norway and Switzerland initially participated in this study.

\section{Inclusion and Exclusion Criteria}

Participants were eligible for inclusion if they had DM2 for 12 years or less, were aged 25 to 60 years, had a $\mathrm{HbA}_{1 \mathrm{c}}$ between $7 \%$ and $10 \%$ inclusive, a BMI between 30 and $40 \mathrm{~kg} / \mathrm{m}^{2}$, and had not responded to a diet or exercise program. The most notable exclusion criteria were type 1 diabetes mellitus, clinically significant hiatal hernia, significant weight loss in the last year ( $>10 \%$ TBL), use of a weight loss drug within the last 3 months or smoking cessation within the last 6 months. Exclusion criteria relevant to diabetes were use of GLP-1 receptor agonists and/or insulin dependence (short-term insulin use during perioperative period was allowed). A full list of inclusion and exclusion criteria have been reported previously ${ }^{[11]}$.

\section{Intervention}

All participants received a Maestro Rechargeable System. The implantation procedure has been described previously in detail ${ }^{[13]}$. Briefly, the two leads of the Maestro Rechargeable System are placed laparoscopically around the anterior and posterior abdominal vagal trunks. The leads are exteriorized after which a rechargeable neuroregulator is connected to the leads and placed in a subcutaneous pocket on the lateral chest wall. Participants recharge the battery of the neuroregulator transcutaneously with a mobile charger and transmit coil every few days for about 30 minutes.

The neuroregulator was programmed to deliver 5000 $\mathrm{Hz}$ with a current amplitude of 3 to $8 \mathrm{~mA}$ for at least 12 hours each day. The therapy amplitude goal was $6 \mathrm{~mA}$. Device parameters (i.e., amplitude and hours of therapy delivered per day) were adjusted if needed to minimize adverse events related to sensations of therapy and maximize weight loss. 


\section{Visit Schedule and data collection}

Participants were seen every week in the first month, every other week through month 3, and then monthly in the first year. Between years one and two participants were seen every other month and then quarterly between the second and third year. No specific diets or exercise regimens were prescribed prior to implant or during the study. However, at each visit participants received 15 minute individual weight management counseling sessions, which consisted of education on strategies for weight loss through exercise, healthy eating and goal tracking. Adverse events, weight and medication changes were recorded at every visit. Glycemic parameters and triplicate blood pressure values were measured at baseline, 1, 4, and 12 weeks, and 6, 12, 18, 24, 30 and 36 months. Waist circumference at the iliac crest (NHANES III Protocol) was measured at baseline and annually. Only the patient's primary care physician made decisions to change any medication regimen.

\section{Statistical Analysis}

Descriptive statistics are used to summarize baseline demographics and medical history information. Means and standard deviations or confidence intervals are used to summarize continuous variables and frequencies and percentages are used to summarize categorical variables.

Longitudinal mixed-effects regression models with unstructured covariance structures and random intercepts for each subject are used to assess changes in continuous parameters. Time was treated categorically in the model so as not to impose a specific functional form to the trajectory over time. Importantly, all observed values were included in the mixed models ${ }^{[14]}$. Analyses of the clinical data were performed in SAS version 9.3. Weight loss was assessed using two methods: 1) Percentage of excess weight loss $(\% \mathrm{EWL})$, which is calculated as: $\% \mathrm{EWL}=$ $100 \%$ * [weight loss / excess body weight at implant (using BMI $25 \mathrm{~kg} / \mathrm{m}^{2}$ as normal)] and 2) Percentage of total body weight loss (\%TBL).

\section{Results}

\section{Subject Demographics and Disposition}

Twenty-eight participants were enrolled in the study and all received a Maestro Rechargeable System. Their mean age was $51 \pm 9$ years, their mean BMI was $37 \pm 3 \mathrm{~kg} / \mathrm{m}^{2}$ and 17 participants were female (61\%). At three years, 22 participants $(79 \%)$ remained enrolled in the study. Eighteen participants (64\%) attended the three-year visit.

Sixteen participants were on metformin only at baseline. Seven other participants were on metformin in combination with other diabetes medications (such as sulfonylureas and thiazolidinediones) and 3 were on other diabetes medications without metformin. Two participants were on no baseline diabetes medications.

\section{Weight Loss}

The weight loss over time is shown in Figure 1. At three years, weight loss was comparable to that at one and two years with an estimated mean $\%$ EWL of $21 \%(95 \% \mathrm{CI}, 14$ to 28$)$ or $7 \%$ TBL (95\% CI, 5 to 9). For those participants who presented for the 3 year visit, the mean $\%$ EWL was $24 \pm 17 \%$ (SD) or $8 \pm$ $6 \%$ TBL. Finally, $61 \%, 39 \%$ and $28 \%$ of participants achieved at least $5 \%, 7.5 \%$ and $10 \%$ TBL, respectively.

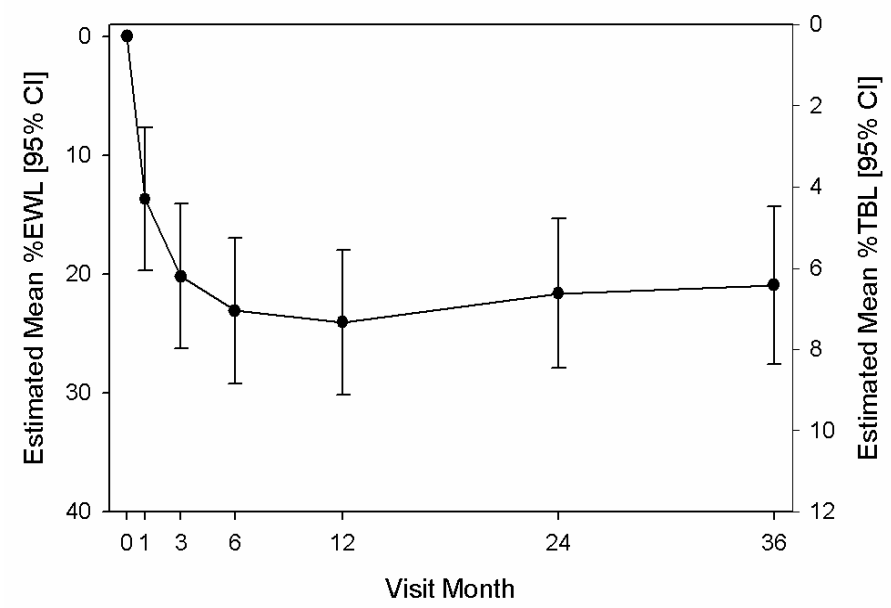

Figure 1: Estimated mean \%EWL and \%TWL and 95\% CI through 3 years.

\section{Glycemic Control}

The changes in HbAlc were from a baseline of $7.8 \% \pm$ $1.1 \%$. Mean reductions in $\mathrm{HbA}_{1 \mathrm{c}}$ over time are shown in Figure 2. As prior reports demonstrated ${ }^{[11,12]}$, the mean estimated $\mathrm{HbA}_{1 \mathrm{c}}$ was significantly reduced by 1.0 percentage point $(95 \% \mathrm{CI}, 0.7$ to 1.4 ) at one year post vBloc therapy initiation. The reduction was 0.6 percentage points $(95 \% \mathrm{CI}, 0.2$ to 1.0$)$ by 2 years, which remained unchanged through three years (Figure 2). The proportion of participants with an $\mathrm{HbA}_{1 \mathrm{c}}$ of $7 \%$ or lower improved from $25 \%$ at baseline to $71 \%$ at 3 years; similar to that observed at 1 and 2 years.

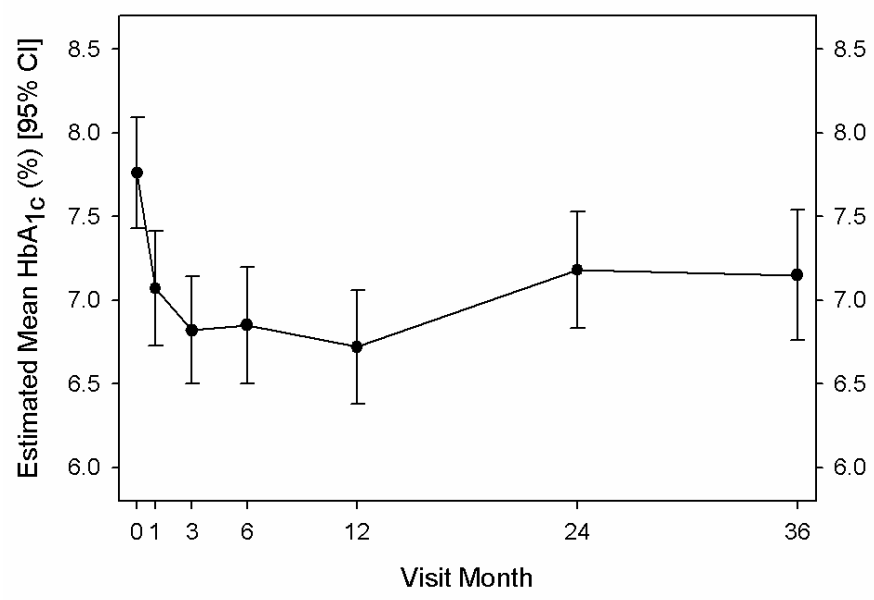

Figure 2: Estimated mean $\mathrm{HbA}_{1 \mathrm{c}}(\%)$ and $95 \% \mathrm{CI}$ through 3 years.

At 3 years, the mean estimated fasting plasma glucose was significantly decreased by $18 \mathrm{mg} / \mathrm{dL}$ (95\% CI, 2 to 34) from $151 \mathrm{mg} / \mathrm{dL}$ at baseline; similar to that reported for reductions at 1 and 2 years following vBloc therapy initiation ${ }^{[11]}$ (Figure 3). 


\section{Vagal Nerve Block in Glycemic Control in Obese Patients}

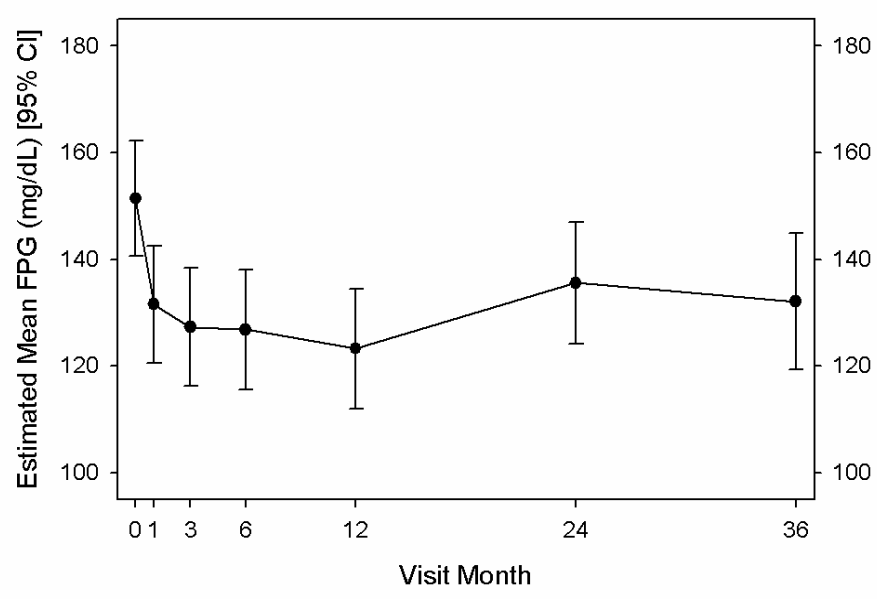

Figure 3: Estimated mean fasting plasma glucose $(\mathrm{mg} / \mathrm{dL})$ and $95 \% \mathrm{CI}$ through 3 years.

\section{Diabetic Medications}

The change in use of diabetes medications from baseline remained relatively constant at 3 years compared to 2 years. Of the 18 participants who attended their 3 year visit, 8 (44\%) had no change in either the number or dosage of medications taken and 7 (39\%) had a decrease in either the number or dosage of medications taken including $4(22 \%)$ of the participants who were able to stop taking diabetes medications. Three (17\%) had an increase in either the number or dosage of medications. None of the participants at the 3 year follow up visit required insulin therapy.

\section{Other Findings of Medical Significance}

At the 3 year visit, there was no significant change in mean systolic and diastolic blood pressure from baseline. A significant decrease in waist circumference was demonstrated at 3 years with a mean reduction of $6.0 \mathrm{~cm}(95 \% \mathrm{CI}, 3$ to 9$)$ from a baseline of $120 \mathrm{~cm}$.

\section{Adverse Events and Surgical Interventions}

As reported previously, there were two SAEs through 24 months $^{[11]}$. There was one additional SAE reported between 2 and 3 years due to pain at the neuroregulator site. The event was considered serious because it resulted in overnight hospitalization following an elective device explant procedure. The SAE resolved following the explant of the device. Information regarding adverse events (AEs) through 2 years has been reported previously ${ }^{[11]}$. The most frequently occurring were mild to moderate heartburn, constipation, and neuroregulator site pain. Between 2 and 3 years there was one additional AE related to moderate heartburn.

No deaths, unanticipated adverse device effects, or life-threatening complications occurred through 36 months. Two surgical revisions occurred through 2 years, as reported previously ${ }^{[11]}$. No additional revisions were required between 2 and 3 years. Six participants withdrew from the study prior to the 3 year follow up visit. Four withdrawals were due to subject decision, one lost to follow up, and one due to adverse event. The subject withdrawal due to adverse event was for pain at the neuroregulator site which resolved following explant of the device.

\section{Discussion}

This investigation demonstrated that intermittent vagal nerve blockade (vBloc) in participants with obesity and type 2 diabetes mellitus resulted in sustained, medically meaningful weight loss and glycemic control through 3 years. The average $\%$ EWL was $21 \%$ at 3 years. $\mathrm{HbA}_{1 \mathrm{c}}$ was reduced 0.6 percentage points at 3 years; a clinically meaningful reduction that resulted in $39 \%$ of participants either reducing or stopping their diabetes medications and $44 \%$ of participants needing no additional diabetes medications. The majority $(71 \%)$ of participants were at or below the American Diabetes Association target for $\mathrm{HbA}_{1 \mathrm{c}}$ of $7 \%$ for people with diabetes. A waist circumference reduction of $6.0 \mathrm{~cm}$ compared to baseline was also observed at 3 years. The safety of vBloc continues to be promising with no life-threatening complications or deaths observed in the study.

Type 2 diabetes mellitus and obesity are two of the most serious societal health concerns throughout the world. Since 1987, the proportion of people with a BMI greater than 30, 40 and $50 \mathrm{~kg} / \mathrm{m}^{2}$ was reported to have increased by approximately $200 \%, 600 \%$ and $1200 \%$, respectively ${ }^{[15]}$. The Longitudinal Assessment of Bariatric Surgery (LABS) reported that in the U.S., $31 \%$ of patients whose BMI was 40 to $50 \mathrm{~kg} / \mathrm{m}^{2}$ were diabetic while patients with a BMI greater than $60 \mathrm{~kg} / \mathrm{m}^{2}$ had a $42 \%$ prevalence of diabetes ${ }^{[16]}$. Additionally, the co-occurrence of DM2 and obesity significantly increases the risk of hypertension and cardiovascular disease ${ }^{[17]}$ hence increasing morbidity and mortality ${ }^{[18]}$. It's estimated that the average annual medical costs for individuals with DM2 adds up to $\$ 13,700^{[19]}$. The American Diabetes Association estimated that DM2 and its complications cost the US health care system 245 billion dollars in 2012 ${ }^{[19]}$.

Patients with DM2 have shown improvements with a decrease in body weight of as little as $5 \%{ }^{[20,21]}$. While a $5 \%$ reduction in body weight can be achieved by non-surgical means, the long term results are often confounded by recidivism and poor patient compliance. The present study findings that the majority of participant's $\mathrm{HbA}_{1 \mathrm{c}}$ remained significantly reduced without the need for additional diabetic medications through 3 years highlights that the amount of weight loss achieved with vBloc therapy helped to slow the progression of diabetes.

There is a large unmet need for effective therapies that are safer and less complicated than conventional bariatric surgical procedures. Potential for serious complications, long-term sequelae, and permanent alterations to the gastrointestinal tract make many potential surgical candidates unwilling to proceed with surgery ${ }^{[6,22,23]}$. The average weight loss achieved in this study was lower than results routinely reported with gastric bypass and sleeve gastrectomy procedures ${ }^{[6,22,23]}$. However, vBloc therapy has demonstrated less complications and a medically impactful weight $\operatorname{loss}^{[10,21]}$. The current results of the VBLOC DM2 investigation support the notion that the benefits of vBloc therapy are largely sustained to 3 years. The ongoing efficacy and safety of vBloc therapy suggests it would likely be attractive to many of the patients who traditionally have not accepted conventional weight loss surgery.

Mono- or poly-diabetic medication regimens have been shown to be an effective tool to improve glycemic control in patients with DM2. However, many patients with DM2 have shown lack of adherence ${ }^{[24]}$ to medication regimens and there is a large cost of prolonged medication use. This study demon- 
strated a decrease in the total number of medications prescribed, with $39 \%$ of participants achieving either a reduction in diabetes medication or no diabetes medication at 3 years compared to their baseline medications.

At each clinic visit, participants received weight management counseling. This counseling may have played a role in the results. However, there were no specific diet or exercise regimens prescribed and no new counseling material was provided after the first year of the study. In addition, there was a substantial decrease in visit frequency between 2 and 3 years. Despite less visits and counseling, weight loss and improvement in $\mathrm{HbA}_{1 \mathrm{c}}$ were maintained. This suggests that counseling did not play a major role in the results of this study.

The limitations of this investigation were that it did not include a control group and that the sample size was small. However, vBloc therapy resulted in statistically significantly greater weight loss compared to the sham control following the un-blinding in the ReCharge study ${ }^{[9]}$. The weight loss in the VBLOC DM2 study mirrors that of the treatment arm of the ReCharge investigation. This supports the reasonable assumption that the weight loss in this study was greatly dependent on vBloc therapy. Future directions of research needed are a larger, confirmatory study with a prospectively defined hypothesis test and mechanistic studies to better characterize the benefit in this patient population.

\section{Conclusion}

Three-year follow-up of the VBLOC DM2 study among patients with obesity and DM2 demonstrated continued efficacy and safety of vBloc therapy with significant and sustained weight loss and improvement in glycemic control. vBloc therapy continues to be well-tolerated.

Funding: The study was supported by EnteroMedics Inc.

Acknowledgements: We acknowledge Drs. Daniel Bessessen, James Freston, Melissa Martinson, and Frank Moody for participation as members of the Data and Safety Monitoring Board and Clinical Events Committee. We also acknowledge Jody Dew for safety data reporting, Lisa Thackeray for statistical analysis, and Brenda Bachmann, Jane Collins, Radhika Butala, Gretel Young, and Magnus Strommen for study coordination.

Conflicts of Interest: Drs. Shikora, R. Brancatisano, Waataja, and Billington have received support as consultants from EnteroMedics Inc. Dr. Tweden is an employee of EnteroMedics Inc. Drs. Herrera and Pantoja received support for proctoring from EnteroMedics Inc. Drs. Toouli, Kow, and A. Brancatisano have received support for traveling in the past from EnteroMedics Inc. Dr. Billington reports that he is also a consultant for NovoNordisk and has received grant support from Covidien. Drs. Kulseng and Johnsen report no conflicts of interest.

Ethical Approval: All procedures performed in studies involving human participants were in accordance with the ethical standards of the institutional and/or national research committee and with the 1964 Helsinki declaration and its later amendments or comparable ethical standards.

\section{References}

1. Danaei, G., Finucane, M.M., Lu, Y., et al. National, regional, and global trends in fasting plasma glucose and diabetes prevalence since 1980: systematic analysis of health examination surveys and epidemiological studies with 370 country-years and 2.7 million participants. (2011) Lancet 378(9785): 31-40.

2. Boyle, J.P., Honeycutt, A.A., Narayan, K.M., et al. Projection of diabetes burden through 2050: Impact of changing demography and disease prevalence in the U.S. (2001) Diabetes Care 24(11): 1936-1940.

3. Gregg, E.W., Zhuo, X., Cheng, Y.J., et al. Trends in lifetime risk and years of life lost due to diabetes in the USA, 1985-2011: a modelling study. (2014) Lancet Diabetes Endocrinol 2(11): 867-874.

4. Jensen, M.D., Ryan, D.H., Apovian, C.M., et al. 2013 AHA/ACC/ TOS guideline for the management of overweight and obesity in adults: a report of the American College of Cardiology/American Heart Association Task Force on Practice Guidelines and The Obesity Society. (2014) J Am Coll Cardiol 63(25): 2985-3023.

5. Knowler, W.C., Barrett-Connor, E., Fowler, S.E., et al. Reduction in the incidence of type 2 diabetes with lifestyle intervention or metformin. (2002) N Engl J Med 346(6): 393-403.

6. Schauer, P.R., Bhatt, D.L., Kirwan, J.P., et al. Bariatric surgery versus intensive medical therapy for diabetes--3-year outcomes. (2014) N Engl J Med 370(21): 2002-2013.

7. Kral, J.G., Gortz, L.Truncalvagotomy in morbid obesity. (1981) Int J Obes 5(4):431-435.

8. Takahashi, T., Owyang, C. Characterization of vagal pathways mediating gastric accommodation reflex in rats. (1997) J Physiol 504 :479488.

9. Ikramuddin, S., Blackstone, R.P., Brancatisano, A., et al. Effect of reversible intermittent intra-abdominal vagal nerve blockade on morbid obesity: the ReCharge randomized clinical trial. (2014) JAMA 312(9): 915-922.

10. Apovian, C.M., Shah, S.N., Wolfe, B.M., et al. Two-Year Outcomes of Vagal Nerve Blocking (vBloc) for the Treatment of Obesity in the ReCharge Trial. (2017) Obes Surg 27(1): 169-176.

11. Shikora, S.A., Toouli, J., Herrera, M.F., et al. Intermittent Vagal Nerve Block for Improvements in Obesity, Cardiovascular Risk Factors, and Glycemic Control in Patients with Type 2 Diabetes Mellitus: 2-Year Results of the VBLOC DM2 Study. (2016) Obes Surg 26(5): 1021-1028.

12. Shikora, S., Toouli, J., Herrera, M.F., et al. Vagal blocking improves glycemic control and elevated blood pressure in obese subjects with type 2 diabetes mellitus. (2013) J Obes 2013: 245683.

13. Camilleri, M., Toouli, J., Herrera, M.F., et al. Intra-abdominal vagal blocking (VBLOC therapy): clinical results with a new implantable medical device. (2008) Surg 143(6): 723-731.

14. Chakraborty, H., Gu, H. A Mixed Model Approach for Intent-toTreat Analysis in Longitudinal Clinical Trials with Missing Values. (2009) In. Research Triangle Park NC: RTI International.

15. Sturm, R., Hattori, A. Morbid obesity rates continue to rise rapidly in the United States. (2013)Int J Obes (Lond) 37(6): 889-891.

16. Belle, S.H., Chapman, W., Courcoulas, A.P., et al. Relationship of body mass index with demographic and clinical characteristics in the Longitudinal Assessment of Bariatric Surgery (LABS). (2008) Surg Obes Relat Dis 4(4): 474-480.

17. Hubert, H.B., Feinleib, M., McNamara, P.M., et al. Obesity as an independent risk factor for cardiovascular disease: a 26-year follow-up of participants in the Framingham Heart Study. (1983) Circ 67(5): 968977.

18. Lew, E.A., Garfinkel, L. Variations in mortality by weight among 750,000 men and women. (1979) J Chronic Dis 32(8): 563-576.

19. Economic costs of diabetes in the U.S. in 2012. (2013) Diab Care 36(4): 1033-1046.

20. Fujioka, K. Benefits of moderate weight loss in patients with type 2 diabetes. (2010) Diab obes metab 12(3): 186-194. 
21. Stevens, J., Truesdale, K.P., McClain, J.E., et al. The definition of weight maintenance. (2006) Int J Obes (Lond) 30(3): 391-399.

22. Courcoulas, A.P., Belle, S.H., Neiberg, R.H., et al. Three-Year Outcomes of Bariatric Surgery vs Lifestyle Intervention for Type 2 Diabetes Mellitus Treatment: A Randomized Clinical Trial. (2015) JAMA Surg 150(10): 931-940.

23. Mingrone, G., Panunzi, S., De Gaetano, A., et al. Bariatric-metabolic surgery versus conventional medical treatment in obese patients with type 2 diabetes: 5 year follow-up of an open-label, single-centre, randomised controlled trial. (2015) Lancet 386(9997): 964-973.

24. Garcia-Perez, L.E., Alvarez, M., Dilla, T., et al. Adherence to therapies in patients with type 2 diabetes. (2013)Diab Ther 4(2): 175-194.

Ommega Online Publishers

Journal Title: Journal of Diabetes and Obesity (JDO)

Journal Short Name: J diabetes Obes

www.ommegaonline.org
Journal ISSN: 2356-0494

E-mail: diabetes@ommegaonline.com

Website: www.ommegaonline.org 